\title{
Experimental study of the pressure loss in aero-engine air-oil separators
}

\author{
A. Laura Cordes \\ laura.cordes@kit.edu
}

B. Tim Pychynski, C. Corina Schwitzke and D. Hans-Jörg Bauer

Karlsruhe Institut für Technologie (KIT)

Institut für Thermische Strömungsmaschinen (ITS)

Karlsruhe

Germany

\section{A. Thiago P. de Carvalho and B. Hervé P. Morvan \\ Gas Turbine \& Transmission Research Centre \\ Nottingham \\ United Kingdom}

\section{ABSTRACT}

The results of extensive experimental testing of an aero-engine air-oil separator are presented and discussed. The study focuses on the pressure loss of the system. Oil enters the device in the form of dispersed droplets. Subsequently, separation occurs by centrifuging larger droplets towards the outer walls and by film formation at the inner surface of a rotating porous material, namely an open-cell metal foam. The work described here is part of a study led jointly by the Karlsruhe Institute of Technology (KIT) and the University of Nottingham (UNott) within a recent EU project.

The goal of the research is to increase the separation efficiency to mitigate oil consumption and emissions, while keeping the pressure loss as low as possible. The aim is to determine the influencing factors on pressure loss and separation efficiency. With this knowledge, a correlation can eventually be derived. Experiments were conducted for three different separator configurations, one without a metal foam and two with metal foams of different pore sizes. For each configuration, a variety of engine-like conditions of air mass flow rate, rotational speed and droplet size was investigated. The experimental results were used to validate and improve the numerical modelling.

Results for the pressure drop and its dependencies on air mass flow rate and the rotational speed were analysed. It is shown that the swirling flow and the dissipation of angular momentum are the most important contributors to the pressure drop, besides the losses due to friction and dissipation caused by the flow passing the metal foam. It was found that the ratio of the rotor speed and the tangential velocity of the fluid is an important parameter to describe the influence of rotation on the pressure loss. Contrary to expectations, the pressure loss is not necessarily increased with a metal foam installed. 
Keywords: Experimental; oil separation; pressure loss; jet engines; breather

\section{NOMENCLATURE}

$\begin{array}{ll}A_{s} & \text { area of streamtube cross section } \\ C & \text { constant for vortex equation } \\ \dot{m}_{\text {air }} & \text { air mass flow rate } \\ n & \text { exponent for vortex equation } \\ p_{\text {in }} & \text { inlet pressure } \\ p_{\text {out }} & \text { outlet pressure } \\ v & \text { velocity } \\ V & \text { volume } \\ r & \text { radius/radial direction of cylindrical co-ordinate system } \\ z & \text { axial direction of cylindrical co-ordinate system }\end{array}$

\section{Greek Symbols}

$\checkmark$ kinematic viscosity

$\varphi$ tangential direction of cylindrical co-ordinate system

$\rho$ density

$\zeta$ loss coefficient

\section{Indices}

co metal foam of coarser grade

$f \quad$ flow

fi metal foam of finer grade

min minimal value: $\dot{m}_{\text {air }}=20 \mathrm{~g} / \mathrm{s}$

crit critical value

$0 \quad$ initial/inlet value

$1 ; 2 ; 3 \quad$ values at the position indicated

I; II $\quad$ vortex I $\left(\frac{r}{r_{0}}>0.4\right)$; Vortex II $\left(\frac{r}{r_{0}}<0.4\right)$

\subsection{INTRODUCTION}

Aero-engine bearings require a steady supply of oil for lubrication and cooling. Ideally, all of the oil running through the bearings is recycled to minimise the total weight of the oil carried on board. At the same time, the oil is not allowed to enter the engine air system, as it might contribute to pollution of the environment and cabin air. Oil leakage from the bearing chamber into the secondary air system is mainly prevented by applying sealing air to the bearing chamber seals. Oil leaving the bearing, and sealing air entering the chamber, mix and generate a complex two-phase flow consisting of oil film formation and droplets of a large range of different diameters, down to one micrometre and below. The two-phase flow in the bearing chamber and the related heat transfer have been studied by various authors ${ }^{(1-6)}$. Oil and air are scavenged through the scavenge port and vent port, respectively. The rotational speed of the shaft and bearing influence the share of oil leaving with the air ${ }^{(4)}$. The polluted 


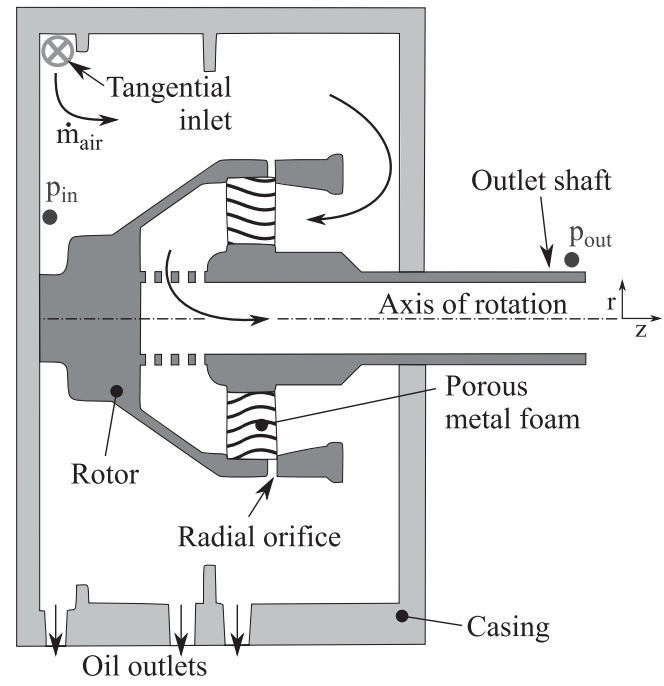

Figure 1. Schematic of a separator. Static and rotating parts indicated in light and dark grey, respectively.

air exiting the bearing chamber through the vent cannot simply be exhausted overboard. This would cause high oil consumption, pollution of the environment and increasing maintenance costs. Therefore, a means to separate the oil from the air is necessary.

Commonly called a breather, the separator consists of a static casing and a rotor, in which a porous medium is installed. A typical breather design, which has been investigated for the present study, is shown in Fig. 1. The air-oil mixture enters the casing tangentially and passes through the rotor and the porous medium. The larger droplets are centrifuged due to the swirl flow in the casing and the smaller droplets are separated by wall adhesion and centrifugal forces in the porous medium. The separated oil is collected in the casing and returned to the oil system. The cleaned air leaves the rotor and is exhausted.

For the breather design, different possibilities have been suggested and studied. Most work is focused on the separation of oil. Gruselle et $\mathrm{al}^{(7)}$ investigated a separator where the airoil mixture is circumferentially accelerated through rotating blades. Results showed that the separation quality depends on the backpressure of the oil outlet, depending on whether the separated oil film was scavenged too fast or too slow. In a further study by Steimes et al ${ }^{(8)}$, an improved prototype was investigated with a metallic foam installed, which lead to an increase in oil separation efficiency. They reported that increasing the oil flow rate leads to a higher absolute oil consumption. They state in a later study ${ }^{(9)}$ that this is likely due to the overall increasing number of droplets, which also implies more small droplets. In the same study, pressure drop across the metallic foam was measured. They showed that the pressure drop increases with the air mass flow rate and depends strongly on the geometry and size of the metallic foam.

Feng et $\mathrm{al}^{(10)}$ investigated a centrifugal separator. They found that the separation efficiency improved when more oil was injected but explained it with the presence of more droplets of a larger diameter.

Sheridan et $\mathrm{al}^{(11)}$ proposed a breather design where a positive pressure difference is created by radially accelerating the flow like an impeller. While the pressure drop results are very promising, it slightly decreases the oil separation efficiency. 


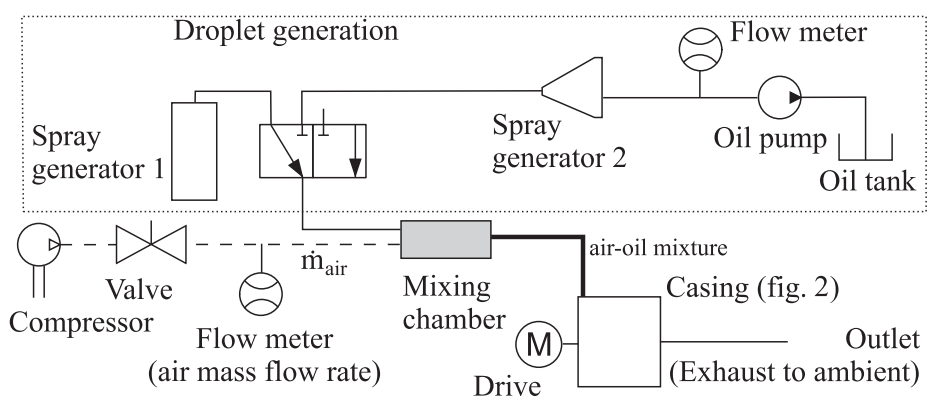

Figure 2. Schematic of the rig set-up.

A configuration similar to the one used in the present study was investigated by Willenborg et $\mathrm{al}^{(12)}$. They found that reducing the air mass flow leads to a decrease of separation efficiency while increasing rotational speed causes it to rise. It was observed that the effect of the breather speed on the pressure drop is stronger than that of the air mass flow rate.

Ideally, the breather exhibits separation efficiencies of $100 \%$ with no pressure loss. While high separation efficiencies of up to $99.8 \%$ have been reported ${ }^{(10,13)}$, the oil, which is not separated, consists mainly of droplets of a diameter of 3-4 $\mu \mathrm{m}$ and below ${ }^{(12)}$.

This research is aimed at deriving design guidelines for reduced pressure loss and improved separation efficiencies. Investigations found in the literature have mostly been dealing with the separation efficiencies, although some improvements regarding the pressure loss have been made $^{(11)}$. The objective of this paper is to investigate and to analyse the main contributors to the pressure drop. This knowledge is crucial for further improvements. Numerical results are used to support and explain the experimental findings. The numerical set-up and results were presented by de Carvalho et $\mathrm{al}^{(14)}$.

First, the experimental set-up is described, followed by a detailed discussion of the pressure loss.

\subsection{EXPERIMENTAL SET-UP}

The rotating test rig consists of an overhung rotor, which is driven by an electric motor up to $7,000 \mathrm{rpm}$. The rotational speed is measured by means of a reflected-light barrier and an electronic tachometer with an accuracy of $0.01 \%$ of the actual value. The casing size enables testing of different rotor designs. However, this study only presents results for the rotor geometry indicated in Fig. 1.

The rig set-up with its air and oil supply is shown in Fig. 2. The test rig is supplied with compressed air up to $10 \mathrm{bar}$ at mass flow rates between 10 and $100 \mathrm{~g} / \mathrm{s}$, which is of the same magnitude as the air mass flow rate leaving the bearing chamber ${ }^{(15)}$. The air mass flow is measured with a hot film probe, which has an accuracy of $\frac{\Delta \dot{m}}{\dot{m}} \leq \pm 3 \%$ over the whole range. Inlet pressure and mass flow rate are controlled using valves, while outlet pressure is close to ambient at all times. The air reaches the mixing chamber at approximately ambient temperature.

Two spray generators are used to represent the smaller droplet spectrum of the air-oilmixtures leaving the bearing chamber. The droplet sizes are smaller than $10 \mu \mathrm{m}$, which has been determined by means of the laser diffraction method. The entering oil mass flow rate can reach values up to $1.81 / \mathrm{h}$, depending on the air mass flow rate. This is due to the design of the 


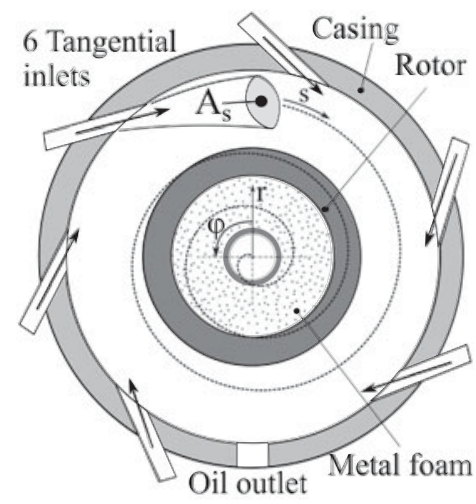

Figure 3. Front view of the separator with an arbitrary projected streamline. Tangential inlets and rotor shown.

chamber where air and oil mix. At low air mass flow rates, up to 50\% of the oil settles there. Oil droplets already separate in the mixing chamber due to gravity and adhesion. This amount is measured and taken into account for the oil mass flow rate.

After the mixing chamber, the air-oil-mixture enters the casing through six tangential inlets with a diameter of $13 \mathrm{~mm}$ each, as indicated in Figs 2 and 3. This inlet configuration creates the tangential velocity component $v_{t, 0}$, which depends on the air mass flow rate. The resulting pre-swirl is supposed to facilitate the separation of large oil droplets through centrifugal effects, as is the case in a cyclone.

Between the casing inlet and exit, the flow has to undergo several $90^{\circ}$ and $180^{\circ}$ turns in the $r$-z-plain (see Fig. 1). The flow enters the outlet shaft through several large holes, which cover two thirds of the shaft circumference. In addition to the flow turns, a porous medium can be placed in the rotor, covering the complete flow path of the air-oil mixture as indicated in Fig. 1. This porous medium is supposed to catch passing oil droplets by wall adhesion and divert them radially outwards to the inner rotor wall, thus improving separation efficiency. In order to prevent separated oil being retained at the outer radius of the porous medium, 16 radial orifices are manufactured into the rotor wall (see Fig. 1). Centrifugal forces cause ejection of the collected oil through these radial orifices. The metal foam is an additional obstacle for the flow. It is therefore expected that it causes the overall pressure loss to rise.

In this study, a metal foam was used as a porous medium and is often referred to as Retimet $^{\circledR}$. Two Retimet ${ }^{\circledR}$ designs with different grades of porosity $\frac{V_{\text {Pores }}}{V_{\text {total }}}$ were used. Overall, pressure losses for three different configurations have been studied:

1. Baseline without a metal foam,

2. With a coarse metal foam of higher porosity and

3. With a fine metal foam of lower porosity.

For each of these three cases, a full factorial test matrix was used varying the air mass flow rate $\dot{m}_{\text {air }}$ and the rotor speed $\omega$. The boundary conditions used are summarised in Table 1 . The baseline case reveals the breather's pressure loss and separation efficiency only due to inertia and centrifugal effects. It is expected that the pressure loss and the separation 


\section{Table 1}

Investigated boundary conditions. A full factorial test matrix was applied

$\begin{array}{lcccccr}\dot{m}_{\text {air }}[\mathrm{g} / \mathrm{s}] & 10 & 20 & 40 & 60 & 80 & 100 \\ \omega[\mathrm{rpm}] & 0^{*} & 2,000 & 4,000 & 6,000 & & \\ \text { *could not be reached at } \dot{m}_{\text {air }}=100 \mathrm{~g} / \mathrm{s} . & & & & \end{array}$

efficiency rise with decreasing porosity of the metal foam. Then, an optimum between those two characteristics needs to be found.

One differential pressure transducer with an accuracy of $0.04 \%$ full scale output, which corresponds to $\pm 40 \mathrm{~Pa}$, was used to quantify the difference between the static inlet pressure $p_{\text {in }}$ at the back plate of the casing and static outlet pressure $p_{\text {out }}$ in a pipe that encloses the rotating shaft at the breather exit, as indicated in Fig. 1. This value is equivalent to the pressure loss due to the loss mechanisms, as will be discussed in the next section. Hence, the pressure loss is defined as:

$$
\Delta p=p_{\text {in }}-p_{\text {out }} .
$$

In order to account for transient effects, the differential pressure is sampled 500 times at a rate of $1 \mathrm{kHz}$ and averaged. The pressure drop measurements were performed once without and once with oil droplets in the system. The difference was within measurement accuracy. Additionally, two absolute pressure sensors were used to measure $p_{\text {in }}$ and $p_{\text {out }}$ independently. Due to their inferior accuracy only the data from the differential pressure transducer was used for the following analysis. The pressure loss was investigated for all three configurations with varying rotational speeds and air mass flow rates.

The data for mass flow rate and pressures are acquired with a peripheral component interconnect device by National Instruments with a 12-bit analog-to-digital conversion.

\subsection{PRESSURE LOSS}

The pressure loss between inlet and outlet is an important breather characteristic, as it affects the pressure and mass flow distribution in the secondary air system and also contributes to overall engine efficiency. The total pressure loss is the result of different contributors:

- Shear forces and thus friction on the wall and within the fluid,

- Pressure loss across the metal foam,

- Pressure loss due to centrifugal effects,

- Work/momentum transferred between the rotor and fluid.

Understanding the significance of the various effects and how they depend on the breather configuration, mass flow rate and rotational rotor speed is important for the improvement of future shaft breather designs with respect to the overall pressure loss. This section deals with the characterisation and analysis of the resulting total pressure loss of the shaft breather design studied experimentally. First, the experimental results are presented and discussed. Second, the observed pressure loss behavior will be analysed and described phenomenologically using simple analytical modelling and Computational Fluid Dynamics (CFD) results obtained 


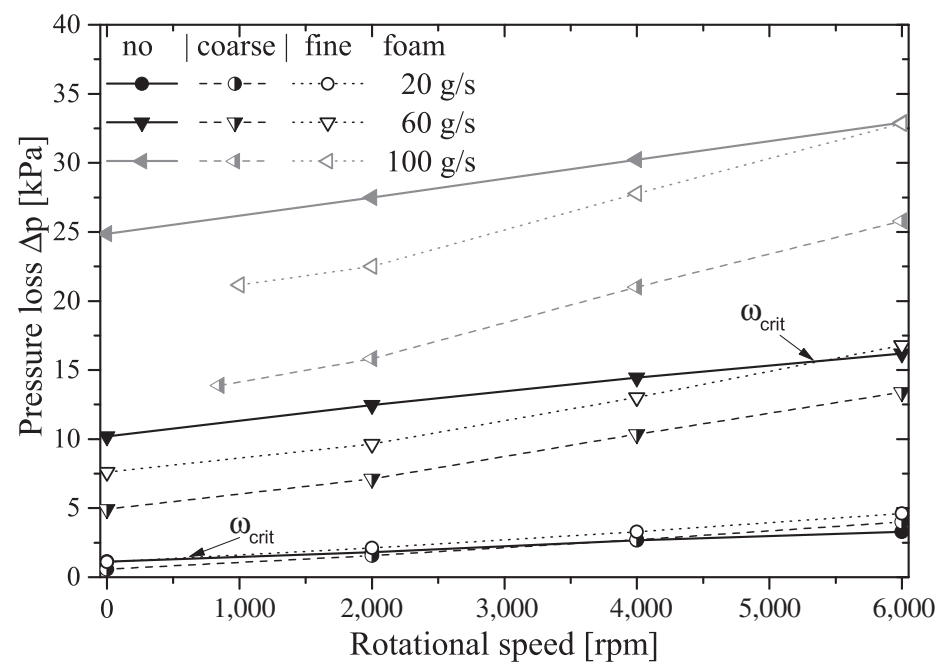

Figure 4. Experimental results for pressure loss $\Delta p$ for $20 \mathrm{~g} / \mathrm{s} \circ, 60 \mathrm{~g} / \mathrm{s} \nabla$ and $100 \mathrm{~g} / \mathrm{s} \triangleleft$ air mass flow. Points connected for visualisation. Critical rotational speeds where losses with a metal foam exceed those with a foam are indicated.

by Carvalho et $\mathrm{al}^{(14)}$. This approach allows both identification and quantification of the dominating effects.

The measured pressure losses across the separator are plotted in Fig. 6 for all three configurations as a function of rotational rotor speed and air mass flow rate. Only selected results are shown in Fig. 6 to facilitate visualisation. For both configurations with a metal foam (coarse and fine), it was not possible to reach zero rotor rotation for high air mass flow rates because the rotor was set into motion by the swirling flow. Figure 6 demonstrates that the pressure drop increases linearly with rotational rotor speed for all three configurations. The slope of the pressure drop increases with increasing mass flow rate. This effect is particularly pronounced for the configurations with a metal foam installed. As expected, the pressure loss is always larger for the configuration with a finer foam than for the coarse foam configuration at the same operating conditions. This difference increases for higher mass flow rates. Surprisingly, however, up to a certain critical rotational speed $\omega_{\text {crit }}$, the pressure loss for the configurations with a metal foam is lower than for the baseline configuration without a metal foam installed. This tendency is reversed for higher rotational speeds. It is interesting to note that this critical rotational speed seems to increase with increasing mass flow rate. Using this effect for application in a breather means that the installation of a metal foam is highly beneficial in terms of pressure loss if the rotational speed stays below the critical speed.

The following analysis is separated into two sections. The first section deals with the analytic description of the pressure loss for the case of zero rotor rotation. This case will allow the explanation and quantification of:

- The lower total pressure losses observed for the configurations with metal foam at low rotational speeds,

- The increasing effect of the mass flow rate on pressure loss. 


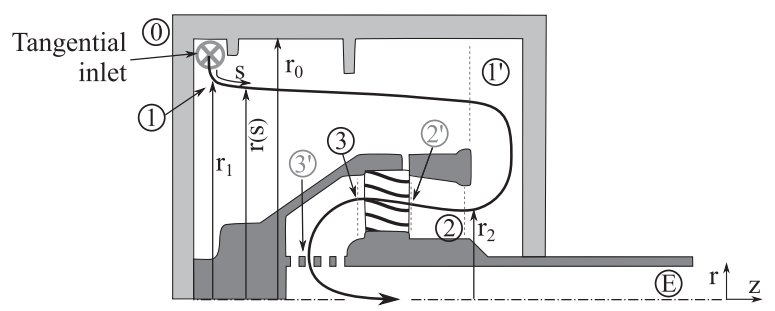

Figure 5. Projected arbitrary streamline through the separator with evaluated positions indicated. Two radii $r_{1}$ and $r_{2}$ sketched exemplarily.

In the second section, the effect of rotational speed will be explained phenomenologically based on the CFD results and on the analytical model derived in the first section.

\subsection{Non-rotating case}

First, the equations used to describe the flow are introduced. Using those with the geometry information, an analytical description of the tangential velocity $v_{\varphi}=f(r)$ is derived. Using these results along with numerical data, a detailed discussion of the phenomena occurring in the system is performed. Then, the effects of a metal foam installed in the rotor are discussed and described analytically.

A projected typical flow path through the breather is indicated in Fig. 5. In reality, the flow follows a helical path, even in the case without rotor rotation, as the flow enters through tangential inlets (comp. Fig. 3). As the swirling fluid is deflected towards a smaller radius, the tangential velocity $v_{\varphi}$ increases according to the conservation of specific angular momentum $h$ :

$$
h=A_{s} v_{\varphi} r=\text { const } .
$$

$A_{S}$ corresponds to the cross section of the stream tube perpendicular to the tangential velocity as defined in Fig. 3. The tangential velocity can be expressed by a vortex equation

$$
v_{\varphi}(r)=\frac{C}{r^{n}},
$$

which is typical for the flow in a cyclone ${ }^{(16)}$. The corresponding change in static pressure between two different radii $r_{1}$ and $r_{2}$ can be determined using the momentum equation in radial direction. Under the assumption that the radial velocity is negligible, it remains only the pressure change due to centrifugal effects:

$$
\Delta p_{v}=\int_{r_{1}}^{r_{2}} \rho \frac{\left(v_{\varphi}(r)\right)^{2}}{r} \mathrm{~d} r=\int_{r_{1}}^{r_{2}} \rho \frac{C^{2}}{r^{2 n+1}} \mathrm{~d} r
$$

The evolution of the streamtube cross section $A_{s}$ along a radius $r$ can be estimated based on the inlet conditions, test rig geometry and CFD results. For this purpose, an arbitrary streamline was investigated, and the streamtube cross section $A_{s}$ and the radius $r$ were determined at several representative points, as indicated in Fig. 5. With Equation (2), the tangential velocity $v_{\varphi}$ is then calculated. It becomes clear that two vortices exist, separated by a velocity decrease due to an increase of streamtube cross section $A_{s}$ between points 1 and 1'. Knowing the 
Table 2

Top rows: Radius $r$ and tangential velocity $v_{\varphi}$ at the indicated points for the case without rotation. Bottom rows: Results for the constants $C$ and $n$ for the piecewise definition of $v_{\varphi}(r)$

\begin{tabular}{|c|c|c|c|c|c|c|}
\hline $\begin{array}{l}\text { Point } \\
\text { Vortex }\end{array}$ & \multicolumn{2}{|c|}{ I } & \multicolumn{4}{|c|}{ II } \\
\hline$r$ & $r_{0}$ & $\frac{4}{5} r_{0}$ & $\frac{3}{4} r_{0}$ & $\frac{1}{3} r_{0}$ & $\frac{1}{3} r_{0}^{*}$ & $\frac{1}{8} r_{0}^{*}$ \\
\hline$v_{\varphi}$ & $v_{\varphi 0}$ & $\frac{5}{4} v_{\varphi 0}$ & $\frac{4}{5} v_{\varphi 0}$ & $v_{\varphi 0}$ & $v_{\varphi 0}^{*}$ & $\frac{8}{5} v_{\varphi 0}^{*}$ \\
\hline $\begin{array}{l}\mathrm{C} \\
\mathrm{n}\end{array}$ & & & & $\begin{array}{c}v_{\varphi 0} \\
0.5\end{array}$ & & \\
\hline
\end{tabular}

(a) without metal foam

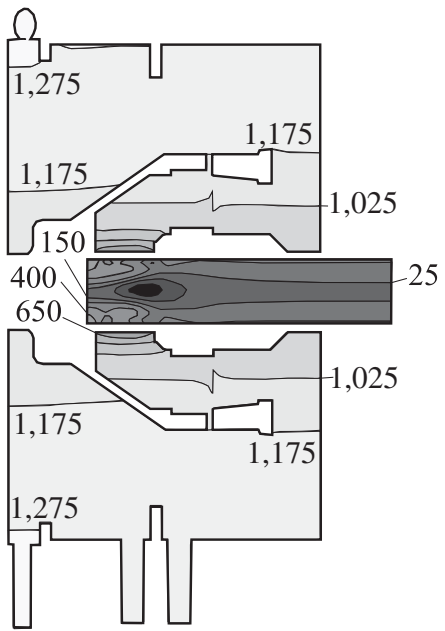

(b) with metal foam

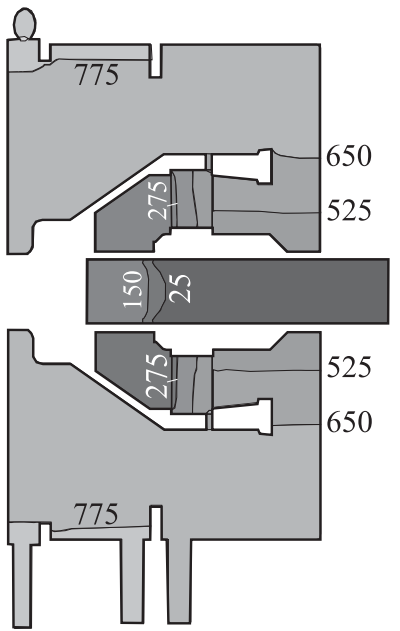

Static pressure $[\mathrm{Pa}]$

$1,400.0$

$1,275.0$

$1,150.0$

$1,025.0$

900.0

775.0

650.0

525.0

400.0

275.0

150.0

25.0

$-100.0$

$-225.0$

Figure 6. Static pressure contour plot for configurations without and with a fine metal foam without rotation at $20 \mathrm{~g} / \mathrm{s}$ air mass flow rate ${ }^{(14)}$. Both outlet shafts have been shortened.

tangential velocity profile, $\mathrm{C}$ and $\mathrm{n}$ from Equation (3) can be identified. The corresponding values of the radius, tangential velocity and of the resulting coefficients $\mathrm{C}$ and $\mathrm{n}$ for the nonrotating test cases are summarised in Table 2 . They were determined assuming incompressible flow conditions, as $\mathrm{Ma}<0.3$ according to the CFD results obtained by de Carvalho et $\mathrm{al}^{(14)}$. Using the respective inlet velocity $v_{\varphi 0}$ as a reference, the values for $C$ and $n$ only depend on the respective inlet conditions.

The static pressure contour plots for zero rotational speed obtained by CFD for both a configuration with and without metal foam insert are shown in Fig. 6 for an air mass flow rate of $20 \mathrm{~g} / \mathrm{s}$. With Equations (2) and (3), and Table 2, the radial development of the tangential velocity $v_{\varphi}$ is plotted in Fig. 7 (flow direction from right to left). The tangential velocity is normalised with the tangential inlet velocity at $20 \mathrm{~g} / \mathrm{s}$ of air mass flow rate $v_{\varphi, 0 \mathrm{~min}}$. 


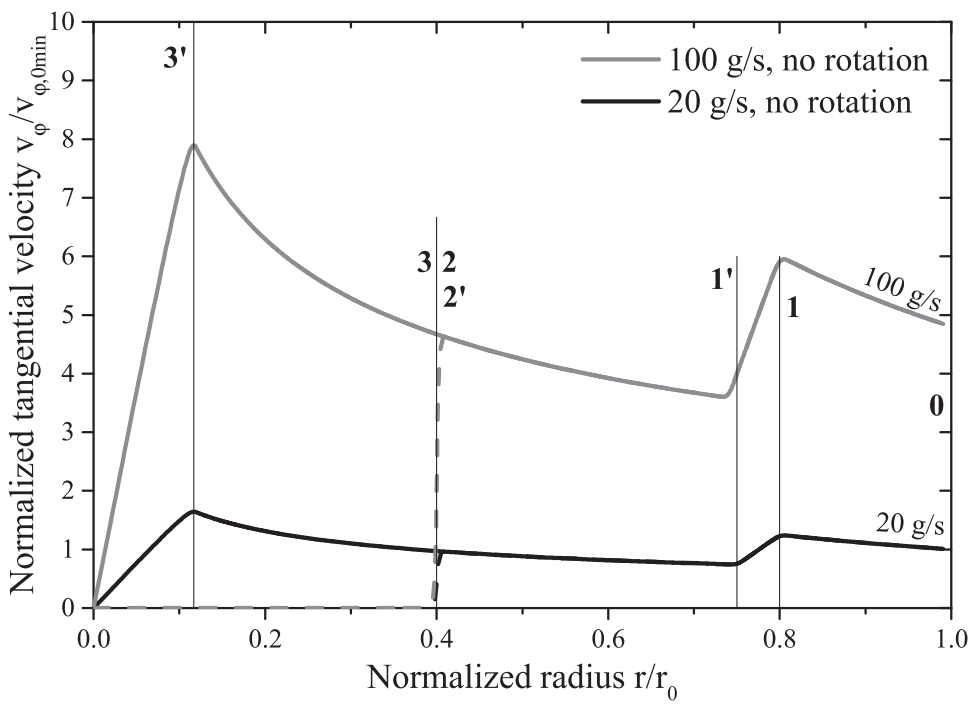

Figure 7. Normalised tangential velocity $v_{\varphi} / v_{\varphi, 0 \min }$ versus the radius $r / r_{0}$ with (dashed lines) and without the metal foam (solid line) without rotation.

For the following discussion, the flow path is divided into several successive sections as indicated in Fig. 5. For the sections between the points 0 and 1, and $1^{\prime}$ and 2, the effect of the decreasing radius dominates and the tangential velocity increases. Even though the radius decreases also slightly between points 1 and $1^{\prime}$, the jet widens, causing the tangential velocity to decrease. If no metal foam is installed, the fluid passes undisturbed through the rotor between points 2 and 3 (along a constant radius). Since the flow is deflected towards a smaller radius between points 3 and $3^{\prime}$, it is once again accelerated. When the flow is deflected to an even smaller radius downstream of point $3^{\prime}$, kinetic energy is dissipated and the tangential flow velocity eventually drops to zero. This is in accordance to the behavior of a Rankine vortex.

For the configurations with metal foam, the tangential flow velocity of the porous medium is imposed onto the fluid. Therefore, the tangential velocity is zero downstream of the metal foam $\left(\frac{r}{r_{0}}<0.4\right.$ in Fig. 7) for the non-rotating case. While the porous medium causes a pressure loss, caused by the axial flow velocity, no further pressure changes occur after the metal foam. This explains why the measured pressure losses for the configurations with metal foam insert are lower than without a foam for zero and low rotational speeds (compare to Fig. 4).

Overall, Fig. 6 shows that the static pressure decreases mainly in the radial direction and across the metal foam. This implies that only the centrifugal effects (comp. Equation (4)) and the losses caused by the metal foam dominate the pressure losses. Therefore, the total pressure loss across the shaft breather is approximated using the following equation:

$$
\Delta p=\underbrace{\rho \int_{r_{0}}^{r_{1^{\prime}}} \frac{\left(v_{\varphi, I}(r)\right)^{2}}{r} \mathrm{~d} r+\rho \int_{r_{1}^{\prime}}^{r_{2}} \frac{\left(v_{\varphi, I I}(r)\right)^{2}}{r} \mathrm{~d} r}_{(a)}+\underbrace{\frac{\rho v_{z}^{2}}{2} \zeta_{f}}_{(b)}+\underbrace{\rho \int_{r_{3}}^{r_{3^{\prime}}} \frac{\left(v_{\varphi, I I^{\prime}}(r)\right)^{2}}{r} \mathrm{~d} r}_{(c)} .
$$




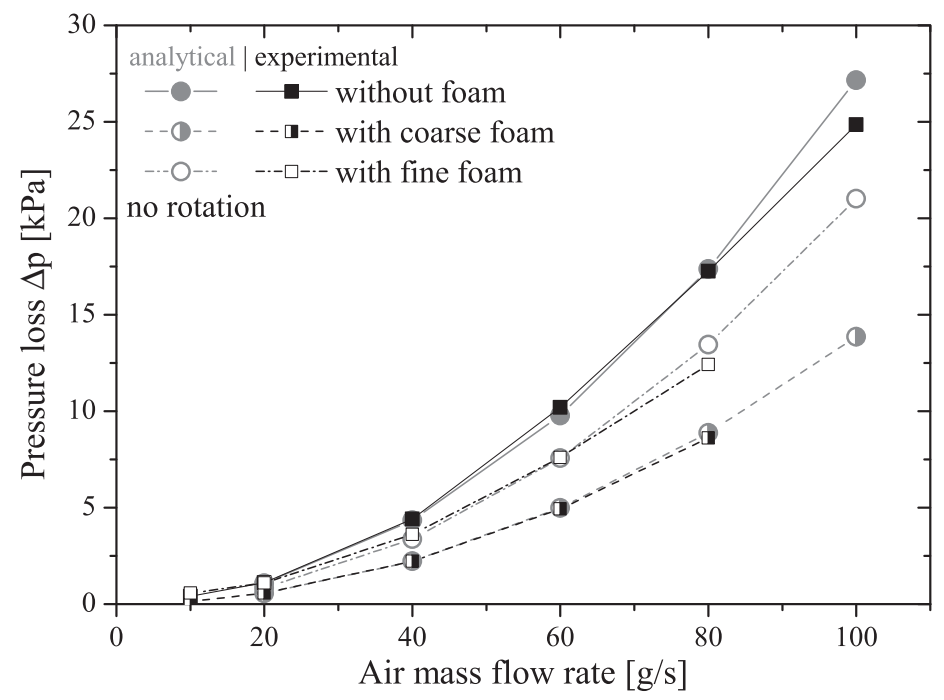

Figure 8. Comparison of the calculated pressure difference (Equation (5)) with the experimental results without rotation. Points connected for visualisation. Deviation between analytical and experimental results less than $10 \%$

The first two terms (a) in Equation (5) give the pressure loss due to centrifugal effects upstream of the metal foam. These terms are identical for all three configurations as discussed previously. The third term (b) represents the pressure loss due to the metal foam where $v_{z}$ corresponds to the axial flow velocity and $\zeta_{f}$ is a loss coefficient, which results from the assumption that the pressure loss depends linearly on the dynamic pressure similar to the losses in a pipe. The loss coefficient is zero for the configuration without foam. The fourth term (c) describes the losses due to centrifugal effects downstream of the foam at point 3. They are zero for the configurations with metal foam (with coarse foam and with fine foam) in the non-rotating case. In case without a foam, $v_{\varphi, I I^{\prime}}(r)=v_{\varphi, I I}(r)$. Equation (5) applies to the rotating case as well, as will be discussed in the next section.

Using the results from the previous discussion of term (a), setting term (c) equal to zero and with the experimental results, the loss coefficients yield to $\zeta_{f, f i}=372$ for the fine metal foam, and $\zeta_{f, c o}=131$ for the coarse metal foam. The resulting overall pressure losses calculated from Equation (5) for the case of zero rotational speed are compared to the experimental data in Fig. 8. The calculated pressure losses show excellent agreement with deviations less than $10 \%$ from the experimental results. This proves that centrifugal effects and pressure loss across the porous medium are indeed the dominating contributing effects.

With the previous analysis, the effects in a non-rotating breather can be summarised: The larger the air mass flow rate, the higher the tangential flow velocities, resulting in much larger pressure drops due to centrifugal effects (see Fig. 7 and Equation (5)). Also, the larger the mass flow rate and hence the axial flow velocity $v_{z}$ through the porous medium, the higher the resulting pressure loss across the metal foam (see term (b) in Equation (5)).

\subsection{Rotating case}

For a rotating rotor and metal foam, similar effects as discussed previously are observed. In addition, work or momentum will be transferred between rotating parts and fluid. If the 


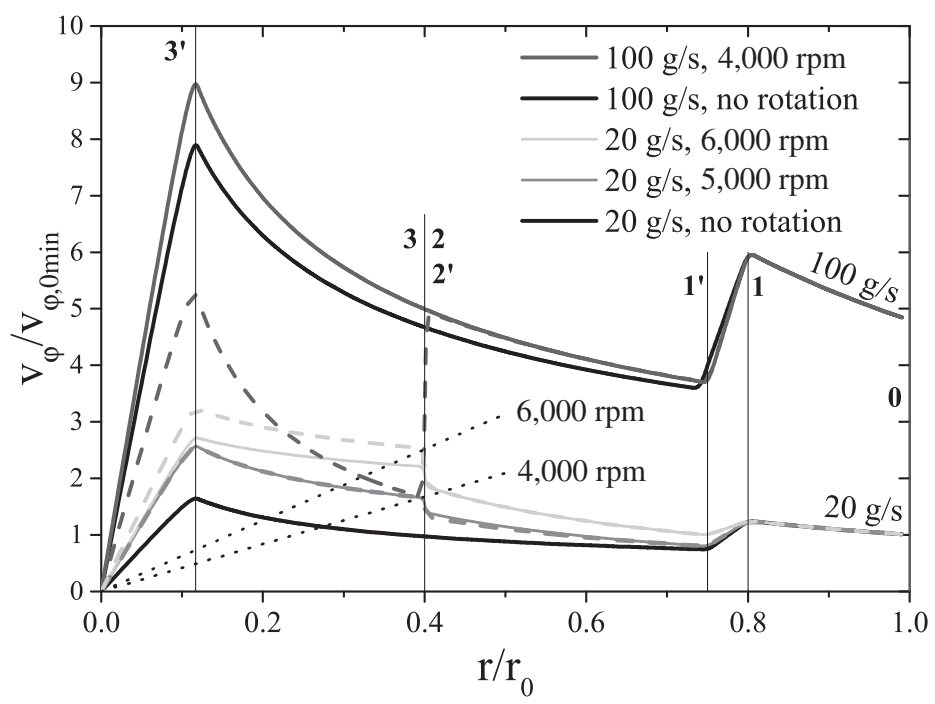

Figure 9. Tangential velocity normalised with the tangential velocity of $\dot{m}_{\text {air }}=20 \mathrm{~g} / \mathrm{s}$ at the inlet $v_{\varphi} / v_{\varphi, 0 \text { min }}$ versus the radius $r / r_{0}$ with (dashed line) and without the metal foam (solid line), and solid body rotation (dotted line).

tangential rotor wall velocity exceeds the tangential flow velocity, the fluid is accelerated and vice versa. The development of the tangential flow velocity along the flow path for different rotational speeds is shown in Fig. 9. The profiles were extracted from the CFD calculations provided by de Carvalho et $\mathrm{al}^{(14)}$. The tangential rotor velocities are plotted as a function of the normalised radius for rotational speeds of $4,000 \mathrm{rpm}$ and $6,000 \mathrm{rpm}$ (dotted lines). The profiles of the tangential velocity without rotation are also shown as reference.

In Fig. 10, the tangential velocity $v_{\varphi}$ of rotor and fluid is normalised with its respective tangential inlet velocity $v_{\varphi 0}$. Without rotation, the normalised curves coincide, as the velocity profile only depends on the inlet velocity.

Figures 9 and 10 support the explanation of the trends observed for the measured pressure losses in Fig. 4. Considering Equation (5), it is clear that the area under the curves in Fig. 9 can be interpreted as a measure of the vortex loss. The smaller the radius, the larger is the contribution to the overall pressure loss.

Upstream of point $2\left(\frac{r}{r_{0}}>0.4\right)$, the rotor rotation leads to slightly higher tangential flow velocities than without rotation, as drag on the flow is reduced compared to the case of zero rotational speed in Fig. 7. It is more pronounced for increasing ratios of $\frac{v_{\text {rotor }}}{v_{\varphi 0}}$ (compare Fig. 10) since the flow will eventually be accelerated. The resulting static pressure drop upstream of the foam increases with the rotational speed (see term (a) in Equation (5)).

If the rotor speed between points 2 and 3 is higher than the tangential velocity of the fluid at that location, an increase in tangential fluid velocity occurs. This is due to the acceleration in tangential direction caused by momentum exchange between the fluid and the rotor. When the rotor is slower than the fluid, this acceleration does not take place. In either case, if a foam is installed, it imposes its own tangential velocity onto the flow.

After point 3, the flow is once again deflected radially inwards towards point $3^{\prime}$. If the rotor rotates and, thus, the flow has a tangential velocity, the tangential velocity will increase and the 


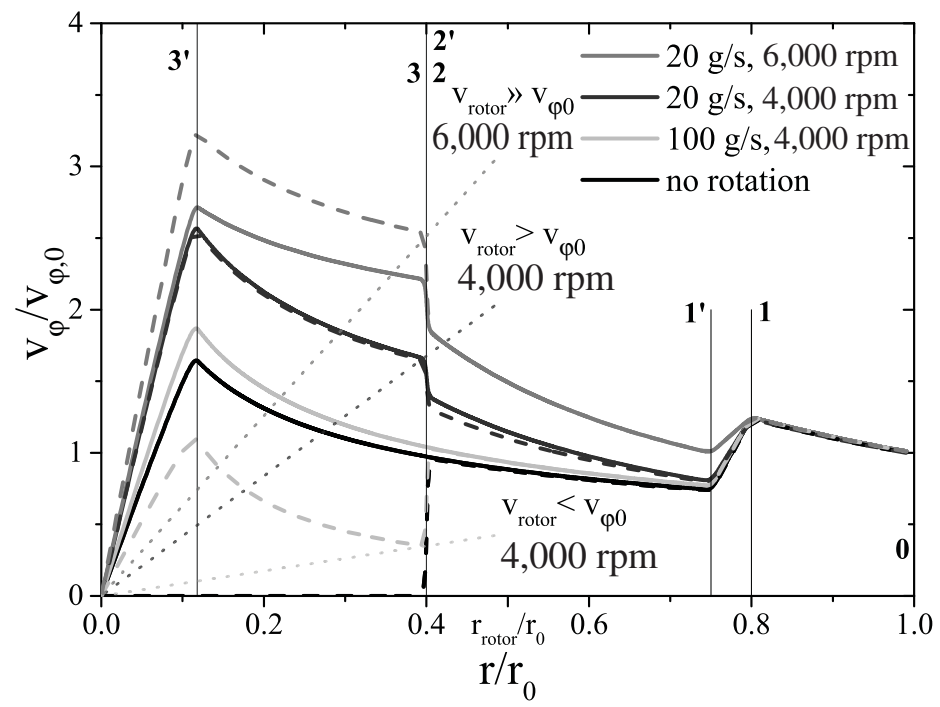

Figure 10. Tangential velocity normalised with its respective tangential velocity at the inlet $v_{\varphi} / v_{\varphi, 0}$ versus the radius $r / r_{0}$ with (dashed line) and without the metal foam (solid line), and solid body rotation (dotted line).

pressure will drop, as described in the previous section. The pressure losses due to centrifugal effects increase with increasing rotational speed as the tangential flow velocities are higher (see Figs 9 and 4).

As can be seen for the case with an air mass flow rate of $20 \mathrm{~g} / \mathrm{s}$ at 4,000 rpm in Fig. 9, if the difference between the fluid's tangential velocity and the foam velocity at point $2^{\prime}$ (directly downstream the foam) is small, the curves with and without a foam show little difference. Therefore, the vortex pressure losses for the configurations with and without foam insert are very similar. For the case with the foam, the additional losses due to the flow through the foam have to be taken into account. This observation places the critical rotor speed lower than 4,000 rpm for this mass flow rate, which can be confirmed with the results in Fig. 4.

For low rotor speeds, the foam causes a much smaller tangential flow velocity downstream from the foam, resulting in significantly lower vortex pressure losses. This can be seen in Fig. 4 for an air mass flow rate of $100 \mathrm{~g} / \mathrm{s}$ at $4,000 \mathrm{rpm}$. Furthermore, if the rotor speed is increased, the flow is accelerated to this higher velocity, resulting in an increased vortex pressure loss for this case (compare $20 \mathrm{~g} / \mathrm{s}$ air mass flow at $6,000 \mathrm{rpm}$ ).

Generally, it can be concluded from Fig. 10 that in the case with a foam installed, if the rotational speed rises, the increase in tangential velocity for $\frac{r}{r_{0}}<0.4$ is significantly higher than in the case without a foam (compare Fig. 10). Thus, the increase in pressure loss due to rising rotational speeds is higher with a foam installed than without a foam, which explains the different slopes in Fig. 4.

The ratio of rotor speed to tangential fluid velocity at the metal foam inlet (point $2^{\prime}$ ) facilitates the explanation of the critical rotational speed beyond which the total pressure losses for a configuration with foam exceeds the total pressure loss without foam. This ratio is therefore an important parameter for describing the phenomena leading to the pressure losses in the breather. 


\subsection{CONCLUSION}

This paper presents the experimental results obtained from a rotating aero-engine oil separator. Pressure drop was recorded for air mass flows rates of up to $100 \mathrm{~g} / \mathrm{s}$, rotational speeds of up to $6,000 \mathrm{rpm}$ and three different configurations, one without a metal foam and two with foams of different porosity.

An analytical approach for the calculation of the pressure losses without rotation has been derived and found to be within excellent agreement with the experimental results. It was proven that the pressure drop is caused mainly by centrifugal forces, resulting from the swirling flow in the separator. Also, significant losses appear when the flow passes the metal foam.

Rotation of the rotor causes the tangential velocities of the fluid to increase and the metal foam imposes its velocity onto the flow. It was shown that the influence of rotation depends on the ratio of the rotor speed to the tangential velocity of the air.

Considering these results for technical applications the pressure loss due to vortex losses can be reduced by decreasing the tangential speed especially close to the axis of rotation. This can be accomplished by installing a metal foam, a flow rectifier or any other device to deflect the flow like guiding vanes or blades. Especially if a rotating metal foam is used in combination with high rotational speed additional measures have to be taken to decrease the tangential velocity before it is dissipated.

\section{REFERENCES}

1. Glahn, A. and Wittig, S. Two-phase air/oil flow in aero engine bearing chambers: Characterization of oil film flows, Journal of Engineering for Gas Turbines and Power, 1996, 118, (3), p 578.

2. Glahn, A., Kurreck, M., Willmann, M. and Wittig, S. Feasibility study on oil droplet flow investigations inside aero engine bearing chambers-PDPA techniques in combination with numerical approaches, Journal of Engineering for Gas Turbines and Power, 1996, 118, (4), p 749, ISSN 07424795.

3. Krug, M.B., Peduto, D., Kurz, W. and BAuER, H.-J. Experimental investigation into the efficiency of an aero-engine oil jet supply system, Journal of Engineering for Gas Turbines and Power, 2015, 137, (1), p 011505. doi: 10.1115/1.4028255.

4. KurZ, W., Dullenkopf, K. and Bauer, H.-J. Influences on the oil split between the offtakes of an aero-engine bearing chamber, ASME Paper No. GT2012-69412, 2012, p 2251.

5. KuRZ, W., Dullenkopf, K. and BAuer, H.-J. Capacitive film thickness measurements in a ventless aero-engine bearing chamber-influence of operating conditions and offtake design, Journal of Engineering for Gas Turbines and Power, 2013, 135, (11), p 112504. doi: 10.1115/1.4025067.

6. Wittig, S., Glahn, A. and Himmelsbach, J. Influence of high rotational speeds on heat transfer and oil film thickness in aero engine bearing chambers, ASME Paper No. 93-GT-209, 1993.

7. Gruselle, F., Steimes, J. and Hendrick, P. Study of a two-phase flow pump and separator system, Journal of Engineering for Gas Turbines and Power, 2011, 133, (6), p 062401. doi:10.1115/1.4002470.

8. Steimes, J., Gruselle, F. and Hendrick, P. Performance study of an air-oil pump and separator solution, ASME Paper No. GT2012-68895, 2012, p 103.

9. Steimes, J., Gruselle, F. and Hendrick, P. Study of an air-oil pump and separator solution for aero engine lubrication systems, ASME Paper No. GT2013-94483, 2013, p V002T01A008.

10. Feng, J., Chang, Y., Peng, X. and Qu, Z. Investigation of the oil-gas separation in a horizontal separator for oil-injected compressor units, Proceedings of the Institution of Mechanical Engineers, Part A: Journal of Power and Energy, 2008, 222, (4), pp 403-412.

11. Sheridan, W.G., Swayze, S.T. and Glahn, J.A. De-oiler system for improved oil containment, ASME Paper No. GT2006-90035, 2006, pp 1283-1290. 
12. Willenborg, K., Klingsporn, M., Tebby, S., Ratcliffe, T., Gorse, P., Dullenkopf, K. and WiTTIG, S. Experimental analysis of air-oil separator performance, Journal of Engineering for Gas Turbines and Power, 2008, 130, (6), ISSN 07424795.

13. KLIngSPORN, M. Advanced transmission and oil system concepts for modern aero-engines, ASME Paper No. GT2004-53578, 2004, pp 391-398.

14. De Carvalho, Thiago, P., Morvan, H.P., Hargreaves, D., Cordes, L. and Höfler, C. Numerical modelling of two-phase flow in aero-engine air/oil separators, ASME Paper No. GT2016-56633, 2016.

15. Farrall, M., Hibberd, S., Simmons, K. and Giddings, D. Prediction of air/oil exit flows in a commercial aero-engine bearing chamber, Proceedings of the Institution of Mechanical Engineers, Part G: Journal of Aerospace Engineering, 2006, 220, (3), pp 197-202.

16. Cortes, C. and GiL, A. Modeling the gas and particle flow inside cyclone separators, Progress in Energy and Combustion Science, 2007, 33, (5), pp 409-452. 